\title{
La dimension relationnelle des communications
}

Étude d'interactions homme-machine tutorielles a-didactiques

The relational dimension of communications: A study of non-didactic tutorial human-computer interactions

Valérie Saint-Dizier de Almeida

\section{OpenEdition}

Journals

Édition électronique

URL : http://journals.openedition.org/activites/2234

DOI : 10.4000/activites.2234

ISSN : $1765-2723$

Éditeur

ARPACT - Association Recherches et Pratiques sur les ACTivités

\section{Référence électronique}

Valérie Saint-Dizier de Almeida, «La dimension relationnelle des communications », Activités [En ligne], 6-2 I octobre 2009, mis en ligne le 15 octobre 2009, consulté le 23 avril 2019. URL : http:// journals.openedition.org/activites/2234 ; DOI : 10.4000/activites.2234

\section{(c) (i) () $\Theta$}

Activités est mis à disposition selon les termes de la licence Creative Commons Attribution - Pas d'Utilisation Commerciale - Pas de Modification 4.0 International. 


\title{
La dimension relationnelle des communications : Étude d'interactions homme-machine tutorielles a-didactiques.
}

\author{
Valérie Saint-Dizier de Almeida \\ Groupe de Recherche sur les Communications \\ Laboratoire InterPsy, EA 4432 - Nancy Université \\ Université Nancy2 BP 33-97 F- 54015 Nancy cedex \\ sdizier@univ-nancy2.fr
}

\begin{abstract}
The relational dimension of communications : A study of non-didactic tutorial human-computer interactions. This paper deals with the sociorelational dimension of communicative interactions. It provides a theoretical and methodological framework for the study of the social dimension of nondidactic tutorial human-computer speech interactions, using the Wizard of $\mathrm{Oz}$ technique. The conversation analysis proceeds within the theoretical framework of Interlocutionary Logic, as developed by Trognon in the 1990s. The social dimension is approached through notions borrowed from social psychology and interactionist linguistics, such as communication contract, role and social position. In this study, one of the main interests of the interlocutionary analysis has been to identify the linguistic means by which protagonists fulfil their roles and jointly build a communication contract.
\end{abstract}

KEYWORDS

Communication, activity, Wizard of $\mathrm{Oz}$, human-computer interaction, tutorial interaction, social relation, communication contract, role, social position, interlocutionary analysis

\section{1.- Introduction}

Toute communication se déploie sur deux dimensions, une dimension opératoire ou technique et une dimension relationnelle (Watzlawick, Beavin, \& Jackson, 1972). Ainsi, les interactions participent de manière simultanée d'une part à la construction d'un sens qui s'inscrit dans la sphère opératoire et d'autre part à la construction de relations sociales et d'images identitaires (Vion, 1992). On peut alors envisager tout énoncé comme contribuant au façonnement d'un sens opératoire et d'un sens relationnel (Cahour, 2006). Partant, comprendre la dynamique des activités qui opèrent dans et au moyen de l'usage du langage suppose que la dimension relationnelle des communications soit investie au même titre que la dimension opératoire.

Dans cet article, nous proposons d'investir cette dimension relationnelle à partir d'une étude de cas. En nous inspirant du cadre développé en psychologie ergonomique pour investir la dimension opératoire et en empruntant des notions et concepts à la linguistique interactionniste et à la psychologie sociale, nous proposons un cadre théorique et méthodologique pour l'étude de la dimension relationnelle d'interactions homme-machine tutorielles a-didactiques obtenues par la technique du magicien d'Oz. Dans notre approche, le contrat de communication est une notion clef. Nous distinguons le contrat de communication externe qui renvoie à l'aspect normatif, institutionnel de la relation existant hors situation d'interaction, du contrat de communication coconstruit qui renvoie à la relation telle qu'elle s'est façonnée pour le cours de l'interaction. Pour instruire le contrat de communication coconstruit nous avons notamment recours aux notions de rôles, rapport de places et de positions.

Dans une première partie, nous rendons compte de la façon dont la dimension relationnelle a été investie dans le champ de la psychologie ergonomique - champ dans lequel nous nous inscrivons. 
Dans une deuxième partie, nous développons le cadre théorique et méthodologique développé pour notre étude. La troisième partie est consacrée à l'étude de cas.

\section{2.- La dimension relationnelle en psychologie ergonomique}

\section{1.- Les rôles}

La dimension relationnelle d'une communication peut être vue à travers les rôles occupés par les différents partenaires. Les premiers travaux en psychologie ergonomique portant sur les communications fonctionnelles, même si cela n'était pas revendiqué explicitement, ont investi cette dimension; certes, pas dans sa dynamique, mais comme une variable indépendante. Il s'agissait après avoir opéré une analyse de contenu de corpus, d'apprécier comment les actes dotés d'une fonction opératoire se distribuaient sur les opérateurs relativement à leur statut. Cette pratique a permis alors de cerner les rôles occupés par les différents opérateurs en situation de travail. Fin des années 90 avec notamment Rogalski (1998), cette variable relationnelle est intégrée au processus d'analyse afin d'étudier le processus de coordination à l'œuvre dans les collectifs de travail : pour chaque propos tenu, il s'agit de préciser qui dit quoi.

Cette détermination des rôles qui consiste à combiner le statut des participants et la fonction opératoire des actions qu'ils réalisent, permet donc d'instruire la dimension relationnelle de l'activité. Cette approche de la relation est utilisée par Valléry (2004) pour l'étude des relations de service; il la complète par une analyse plus fine des interactions qui s'y déploient. Cahour (2002) l'utilise pour l'étude des activités collaboratives de conception en présentiel; Barcellini, Détienne et Burkhardt (2007), pour l'étude des activités collaboratives de conception médiatisée via les NTIC.

\section{2.- La qualité relationnelle}

Dans le champ des relations de service, Falzon et Cerf (2005) ont introduit la notion de «qualité relationnelle ». On s'oriente alors vers l'étude de certaines séquences: celles qui comportent des marques de civilités (politesse, rituels d'ouverture, de fermeture), celles par lesquelles sont exprimées des émotions, celles au cours desquelles apparaissent des problèmes, des conflits ou qui sont susceptibles d'en générer.

Notons que dans les années 80-90, les chercheurs travaillant sur les communications de travail excluaient ce type de séquences du processus d'analyse, considérant qu'elles ne faisaient pas partie des communications fonctionnelles (Grusenmeyer, 1996). Quoi qu'il en soit, elles sont constitutives des activités professionnelles qui opèrent en situation d'interaction langagière et sont maintenant intégrées au processus d'analyse. Les travaux par exemple de Boucheix et Ménard (2000 in Valléry, Boucheix, Leduc, \& Cerf, 2005) illustrent cette visée intégratrice. Ils montrent que les actions produites en situation de service d'aides à la personne peuvent se distribuer sur deux catégories fonctionnelles, celles ayant une fonction de régulation sociale (dimension qui nous intéresse ici) et celles ayant une fonction de régulation de l'action. Il apparaît clairement dans ce type d'étude que ces deux fonctions sont constitutives de l'activité et sont considérées comme étant au même niveau - i.e. il n'y en a pas une qui serait subsidiaire par rapport à l'autre.

L'appréhension de la régulation relationnelle peut se faire à partir de l'analyse de séquences extraites de corpus. Par exemple, Boucheix et Ménard (ibid.) retiennent les séquences où apparaissent des remerciements, des salutations, des expressions émotionnelles, des interpellations affectives, etc. Brangier (2002) répertorie des techniques discursives visant à réduire les attitudes revendicatives comme reformuler les inquiétudes, anticiper un conflit et le désamorcer, faire des blagues, plaisanter, etc. Caroly (2000) étudie les rituels d'ouverture, les marques de politesse, les techniques pour anticiper des conflits. 
On peut aussi accéder à des informations sur la régulation relationnelle par le biais des entretiens. Par cette technique, on peut recueillir des informations sur les stratégies utilisées pour établir une relation de confiance (Caroly, 2004), pour dépasser ou gérer des situations problématiques ou difficiles (Caroly, 2004 op.cit.; Djibo, 2008).

Ainsi, la qualité relationnelle d'une communication est appréhendée de manière quelque peu sporadique, via l'étude de séquences (transcrites ou rapportées) ayant une fonction première de régulation sociale et/ou exploitant des ressources appartenant au registre des civilités.

Ce constat trouve une explication dans les propos de Falzon et Cerf (2005 op. cit.). Selon ces auteurs, même une transaction simple et sans difficulté suppose la gestion du niveau relationnel; mais, « le plus souvent, celle-ci ne sera guère visible, puisque ne donnant lieu à aucune négociation particulière ou inhabituelle. En revanche, dès lors que des difficultés spécifiques surgiront (...) des sous-dialogues seront mis en œuvre afin d'aplanir ces difficultés » (Falzon, \& Cerf, 2005, pp. 55-56). Ce sont alors ces sous-dialogues auxquels est affectée une fonction de régulation relationnelle, qui sont particulièrement investis par les chercheurs travaillant sur la qualité relationnelle.

\section{3.- La distance relationnelle}

Mentionnons également les travaux des chercheurs dans le champ de l'ergonomie des interfaces, qui, en cherchant à cerner les singularités du dialogue homme-machine (DHM) comparé au dialogue homme-homme, ont investi un versant de la relation que Marcoccia (2007) dénomme: l'axe de la relation horizontale. Cet axe de la relation renvoie au degré de proximité/distance entre les interlocuteurs.

Les caractéristiques rapportées dans le champ du DHM comme la quasi-absence de rituels d'ouverture, de formules de politesse, le mode d'adressage utilisé, les structures phrastiques simples... (Sperandio, \& Letang-Figeac, 1986; Pierrel, 1987; Bilange, 1992; etc.) constituent des marqueurs de la relation horizontale.

\section{3.- Cadre pour l'étude de la dimension relationnelle}

Soutenant que le versant relationnel mérite d'être appréhendé au même titre que la dimension opératoire, le cadre - son format - que nous utilisons est fortement inspiré de celui développé en psychologie ergonomique pour l'étude de la dimension opératoire des activités finalisées. Plus précisément, nous opérons un parallèle entre «modèle de tâche » et « contrat de communication ».

Nous posons que la notion de contrat de communication est à la relation, ce que le modèle de tâche est à la dimension opératoire.

En effet, le contrat de communication, tout comme le modèle de tâche, peut se décliner en contrat théorique, représenté, actualisé et effectif. Considérons la citation suivante: «l'activité du sujet dépend, partiellement, de ce qu'il se représente devoir être les comportements à adopter dans telle situation concrète, cette activité sera subordonnée à la représentation qu'il se fait des normes valables pour cette situation » (Vion, 1992, p. 75). À travers cette citation, notons tout d'abord que le contrat de communication constitue une ressource (au sens de Suchman, 1987) dans le processus de l'activité, au même titre que le modèle de tâche. Si maintenant nous étudions plus finement cette citation, il est possible d'y cerner la participation des différents types de contrats mentionnés ci-avant:

- «L'activité du sujet» traduit l'accomplissement en acte d'un contrat de communication effectif;

- «Ce qu'il se représente devoir être les comportements à adopter dans telle situation concrète » renvoie au contrat de communication actualisé ;

- «La représentation qu'il se fait des normes valables pour cette situation » renvoie au contrat de communication représenté;

- Les « normes valables pour cette situation » correspondent au contrat de communication 
théorique devant être invoqué dans ce type de situation.

Le schéma ci-après permet de visualiser le parallélisme entre modèle de tâche et contrat de communication.

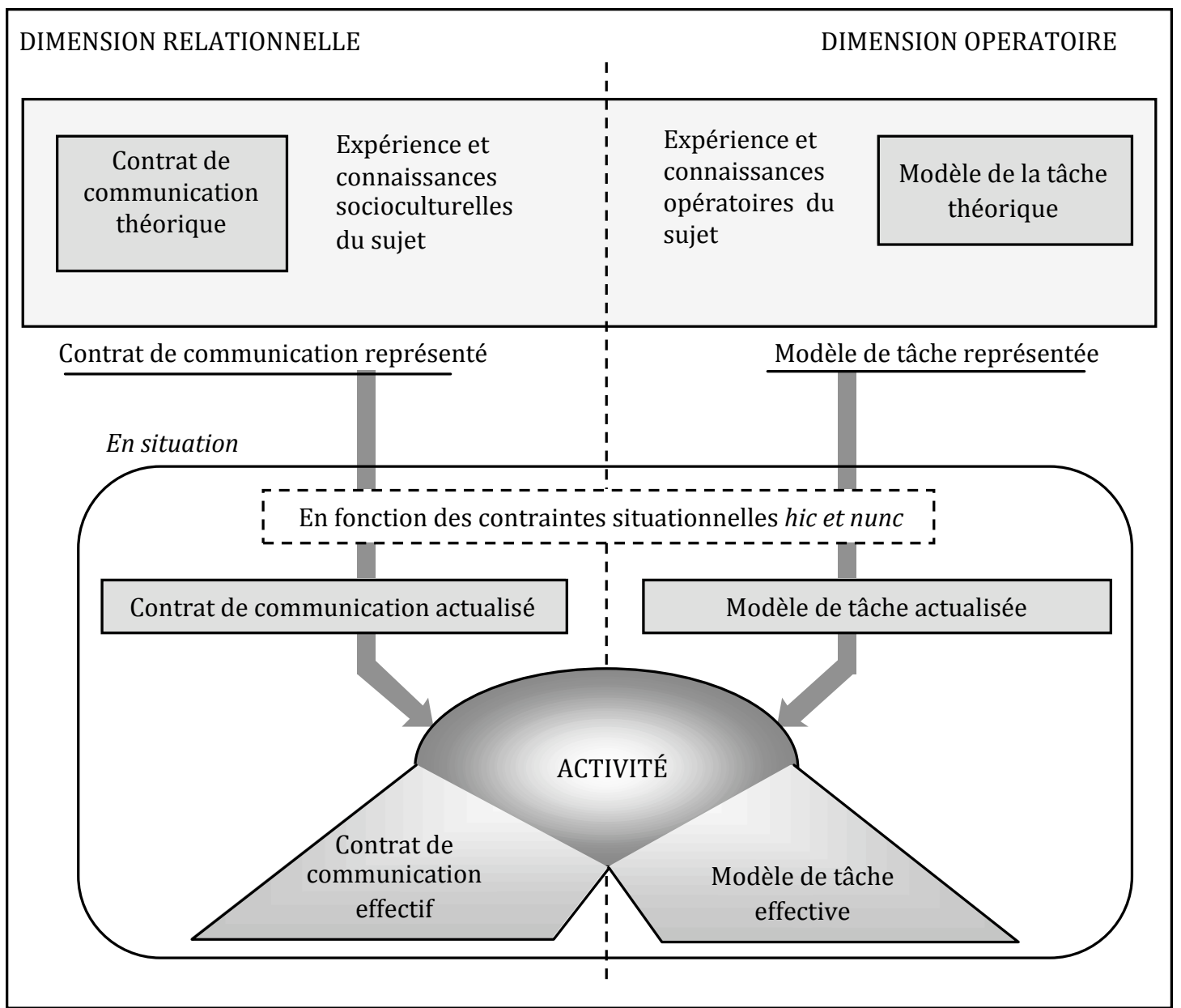

Figure 1: Parallélisme entre le modèle de tâche et le contrat de communication.

Figure 1: Parallelism between the task model and the communication contract

Le contrat de communication théorique peut être qualifié dans la littérature de contrat de communication prescrit, normatif, institutionnel; le contrat de communication effectif, de contrat accompli, façonné, élaboré. Pour notre part, nous dénommerons le premier, « contrat de communication externe » et le second, « contrat de communication coconstruit ».

Pour l'étude de la dimension relationnelle de notre corpus, nous avons recours à ces deux notions. Nous les définissons à la suite.

\section{1.- Le contrat de communication externe}

Si le modèle de tâche prescrite permet à l'opérateur de se faire une idée sur la tâche à résoudre, son état final, les actions, opérations à réaliser, les procédures..., le contrat de communication externe permet à l'agent de se faire une idée sur la nature de la relation qui va se déployer, sur les rôles qui vont être occupés, la façon dont ils vont s'accomplir...

Le contrat de communication externe est donc un élément extérieur à l'interaction. Il a particulièrement été développé par Charaudeau (1983). Il est pré-défini par le contexte social (Bromberg, 2007). Il va dépendre du cadre institutionnel, du statut des membres et de l'enjeu de l'interaction. 
Selon Charaudeau (2006), c'est un élément constitutif de toute communication. Il fournit un cadre pour l'action et pour l'interprétation (Schubauer-Léoni, 1986). Il détermine les modalités d'échange qui sont attendues par les uns et les autres aux différents moments de l'interaction et donc fournit des indications sur les comportements à adopter.

Le contrat de communication externe peut se définir comme un contrat par lequel les participants à une interaction acceptent tacitement un certain nombre de règles et de principes rendant possible l'interaction; il permet à chaque interactant de se faire une idée sur ses droits, ses devoirs et ceux de l'autre (Charaudeau, 1983 op.cit.). Le contrat renseigne sur « ce qui peut être dit ou fait dans une situation donnée, de connaître les objets que l'on peut «mettre en communication » ainsi que la manière de le faire. » (Vion, 1992 op.cit., p. 74).

Ainsi, tout comme la tâche prescrite, le contrat externe va fonctionner comme une ressource dans la dynamique de l'activité. Il va constituer une ressource pour guider l'action et pour interpréter les actions d'autrui. On peut y accéder par des études documentaires, des entretiens auprès des prescripteurs voire des acteurs. Le contrat externe possède les mêmes caractéristiques que le modèle de tâche prescrite; il peut être plus ou moins explicite, plus ou moins accessible.

\section{2.- Le contrat de communication coconstruit}

Si le modèle de tâche effective rend compte de ce qui a été réalisé pour résoudre la tâche, la réalisation successive de sous-buts, les procédures utilisées..., le contrat de communication coconstruit rend compte des rôles réalisés, de la façon dont ils se sont accomplis, de la relation qui s'est façonnée progressivement au cours de l'interaction. La notion de contrat de communication coconstruit est inspirée de l'acception du contrat de communication telle qu'utilisée par Ghiglione (1989) et Camus (1999) - ces chercheurs ont par exemple étudié la façon dont s'opère le passage d'un contrat à un autre dans le cours de l'interaction.

Ainsi, le contrat de communication coconstruit est un objet qui va s'élaborer, se façonner, se construire, s'accomplir dans et au moyen des activités des partenaires ; c'est donc par l'analyse des communications produites qu'il est possible d'y accéder.

Pour instruire le contrat de communication coconstruit, nous retenons :

- Les rôles: qui fait quoi?

— Les moyens langagiers : quels sont les procédés utilisés pour accomplir les rôles?

— L'axe de la relation verticale : quelle est la relation hiérarchique entre les interlocuteurs?

- L'axe de la relation horizontale : quelle est la distance/proximité entre les interlocuteurs?

\subsection{1.- Les rôles}

Identifier les rôles, consiste dans un premier temps à lister les fonctions opératoires réalisées dans la situation d'interaction. Les fonctions sont déterminées à partir de l'analyse du contenu des actes produits et en référence à la tâche à résoudre. Ensuite à chaque acte produit est affectée une fonction opératoire. L'affectation se fait relativement 1) à l'action dans sa dimension descriptive, 2) au but supposé être poursuivi par l'opérateur et à la situation hic et nunc et 3) aux fonctions opératoires disponibles qui ont été identifiées au préalable (Delgoulet, Kerguelen, \& Barthe, 2000).

Déterminer les rôles, revient à préciser pour chaque fonction opératoire identifiée, le statut de la personne qui l'accomplit (statut invoqué dans le cadre de l'interaction).

\subsection{2.- Les procédés langagiers utilisés}

Cerner la manière dont les rôles se sont accompli revient à identifier et à formaliser les procédés langagiers utilisés pour opérer leur réalisation. Aussi, étudier les procédés langagiers ne peut se réduire à décrire le matériau linguistique utilisé en l'isolant de son contexte d'apparition; l'acte doit être décrit relativement aux relations qu'il entretient avec les autres actes inscrits dans son espace séquentiel. 
Pour rendre compte de ces procédés de façon suffisamment générique d'une part et d'autre part déterminer leur fonction interlocutoire, nous avons recours à la logique interlocutoire (Ghiglione, \& Trognon, 1993).

Cette logique satisfait ces deux exigences: les notions de force illocutoire et de contenu propositionnel permettent de décrire l'activité langagière dans un format suffisamment générique; les propriétés qui articulent les conditions de réussite et de satisfaction des actes de langage permettent de restituer les articulations entre les composants du discours et sur cette base de déterminer leur fonction interlocutoire (nous développerons ultérieurement les éléments de la logique interlocutoire utilisés pour l'analyse).

\subsection{3.- L'axe de la relation verticale}

La relation verticale renvoie à la relation hiérarchique qui s'établit entre les participants et se matérialise à travers leurs échanges (Marcoccia, 2007).

Pour investir cette dimension, Kerbrat-Orecchioni (1988) se réfère à la notion de rapport de places: «La notion de place renvoie à l'idée développée entre autres par Flahaut (1978) qu'au cours du déroulement d'une interaction les différents partenaires de l'échange peuvent se trouver positionnés en un lieu différent sur cet axe vertical invisible qui structure leur relation interpersonnelle. On dit alors que l'un d'entre eux se trouve occuper une position « haute » de «dominant », cependant que l'autre est mis en position « basse », de « dominé » (Kerbrat-Orecchioni, 1988, op.cit., p. 185).

Ainsi lorsqu'un individu s'exprime, il occupe une place particulière et convoque simultanément l'autre à une place corrélative. Par exemple, en donnant un ordre à quelqu'un, un locuteur occupe une place de demandeur et convoque son partenaire à une place de sollicité. À ces rapports de places sont associées des positions sociales (hautes versus basses). En reprenant notre exemple, le locuteur lorsqu'il énonce l'ordre, serait en position haute car il se donne le pouvoir de requérir quelque chose de son partenaire; celle du partenaire, basse puisque soumis au désir du premier. Le rapport de places est négociable, ce qui signifie qu'au tour suivant, le sollicité peut inverser le rapport en refusant de satisfaire la demande.

Pour instruire les rapports de places, Kerbrat-Orecchioni propose l'identification de taxèmes qui sont des indicateurs de places - les différents taxèmes identifiés par l'auteur figurent dans (Kerbrat-Orecchioni, 1988, op.cit.).

Identifier la position occupée par chaque partenaire à chaque énonciation n'est pas chose aisée car une même énonciation peut comporter plusieurs taxèmes dont il faut estimer le poids. Toutefois certains taxèmes sont plus puissants que d'autres. Certains dotent l'acte d'une valeur taxémique, les autres accentuent ou atténuent cette valeur. Nous poserons, pour notre étude, que la valeur taxémique principale d'un acte est généralement donnée par la force illocutoire.

Selon les auteurs précédents,

- La force illocutoire directive (qui impose à l'autre de faire ou de dire quelque chose) est un taxème de position haute pour le locuteur et donc basse pour l'allocutaire (puisque l'allocutaire est placé en position de soumission);

- La force illocutoire commissive (par laquelle le locuteur s'engage à faire quelque chose) est un taxème de position basse pour le locuteur (puisque c'est à lui de faire quelque chose) et donc haute pour l'allocutaire;

- Le remerciement dont la force illocutoire est expressive (elle exprime l'état psychologique du locuteur) est un taxème de position basse pour le locuteur car considéré par Kerbrat-Orecchioni (ibid.) comme un acte auto-dégradant.

La valeur taxémique d'un acte peut être atténuée ou accentuée par la présence d'autres taxèmes.

Par exemple, la fonction initiative d'un acte est un taxème de position haute pour le locuteur: il in- 
dique que le locuteur a le pouvoir sur la trame du discours. Ainsi, si un ordre initie une séquence, la valeur taxémique de position haute pour le locuteur sera accentuée et donc corrélativement, celle de l'allocutaire sera encore plus basse.

Les formules de politesse sont des taxèmes de position basse pour le locuteur. Si un acte illocutoire directif comporte une formule de politesse, la valeur taxémique de l'acte sera atténuée; le locuteur sera alors en position moins haute et corrélativement, l'allocutaire en position moins basse.

Pour l'analyse du corpus, nous utiliserons certains des taxèmes identifiés par Kerbrat-Orecchioni (ibid.) et également d'autres que l'analyse aura révélés.

\subsection{4.- L'axe de la relation horizontale}

La relation horizontale renvoie à la proximité ou à la distance qui s'établit entre les interlocuteurs et qui se matérialise à travers leurs échanges (Marcoccia, 2007, op.cit.). Là aussi, il s'agit d'identifier des taxèmes qui sont ici des marqueurs de la relation horizontale. Si Kerbrat-Orecchioni a davantage investi l'axe de la relation verticale, Marcoccia (ibid.) utilise certains des taxèmes qu'elle a identifiés pour instruire l'axe de la relation horizontale. Par exemple, les pronoms d'allocution (tutoiement, vouvoiement,), les titres, les termes d'adresse et autres expressions appellatives sont considérés comme des taxèmes renseignant sur les relations notamment socio-affectives; les choix lexicaux, grammaticaux, syntaxiques... constituent des éléments pouvant instruire le style des échanges (familier, professionnel, intime, distant...) et sont à ce titre des taxèmes de la relation horizontale.

\section{4.- L'étude de cas : la recherche GOCAD}

La communication langagière que nous allons étudier a été produite pour obtenir des données destinées à la conception d'un système d'assistance interactif en langue naturelle. Il s'agissait de doter un logiciel de géologie d'une interface intelligente (recherche GOCAD $\left.{ }^{1}\right)$.

Pour obtenir des communications qui constitueraient une ressource pertinente pour la conception de ce système, nous avons, par la mise en place du dispositif, induit une situation d'interactions hommemachine tutorielles a-didactiques.

Pour obtenir une interaction homme-machine, nous avons utilisé la technique du magicien d'Oz (on fait croire au sujet qu'il interagit avec une machine, alors qu'en réalité il interagit avec un humain chargé de simuler une machine). Par la mise en place du dispositif, nous avons tenté de susciter une interaction tutorielle a-didactique. Une interaction didactique se distingue d'une interaction a-didactique par l'enjeu de l'interaction et les statuts des partenaires. Dans le premier cas, l'enjeu est l'apprentissage. Le détenteur du savoir a un statut d'enseignant; l'autre, un statut d'élève. Dans le second cas, l'enjeu est la résolution - lorsque des connaissances expertes sont communiquées, elles le sont indépendamment d'une intention didactique (Brousseau, 1986). Le détenteur du savoir a un statut d'assistant; l'autre, un statut de résolveur.

Ces caractéristiques conduisent à deux formes de contrats de communication externes, le premier est dit didactique, le second a-didactique (Brousseau, 1986, op.cit.). Dans le cours de l'action, le contrat de communication didactique induit une forte inégalité des positions (Kerbrat-Orecchioni, 1988): l'enseignant est davantage en position haute et l'élève en position basse. Par le choix des consignes, nous tentons d'atténuer cette inégalité en induisant une interaction a-didactique. Il s'agit donc, en reprenant la terminologie de Maïs (1989 cité dans Falzon, \& Pasqualetti, 2000) d'induire une situation

1. La recherche GOCAD s'inscrivait dans un projet interdisciplinaire : le projet IRMA (Interaction Répartie Multimodale pour le MultimédiA) impliquant l'ENSG, l'équipe Dialogue du CRIN/LORIA (URA 262) et le GRC- Université Nancy2. Le projet GOCAD visait la conception d'une interface multimodale intelligente à partir d'une application cible: le logiciel GOCAD (un modeleur de surfaces géologiques développé par l'ENSG). L'objectif était d'implémenter à ce logiciel une interface intégrant un système d'aide en langue naturelle. $C$ 'est à partir des résultats des analyses d'un corpus d'interactions que le système d'assistance a été développé. Pour la modélisation du système, nous renvoyons à Saint-Dizier (1995) et Saint-Dizier de Almeida, (1997); pour son implémentation, à Chapelier (1996). 
d'action - une situation d'usage finalisé par une tâche - et non une situation d'apprentissage.

\section{1.- Le dispositif de l'expérience}

\subsection{1.- La définition des tâches}

Quatre tâches ont été définies avec les concepteurs du logiciel (qui sont des enseignants-chercheurs en géologie) de façon à utiliser un maximum de commandes de l'application. Réaliser une tâche avec le logiciel GOCAD consiste à partir d'un fichier de données à opérer une succession de commandes qui permettent une bonne visualisation d'une ou de plusieurs surfaces géologiques.

La résolution de chaque tâche requiert l'exécution d'une dizaine de commandes; des commandes annexes qui améliorent la visibilité des surfaces peuvent également être effectuées (opérer une rotation, faire un zoom, changer de couleur).

Dans le contexte de travail, pour qu'une commande soit exécutable, il faut qu'elle soit comprise, qu'elle soit complète (ses attributs doivent être fixés dans l'espace de l'interlocution; par exemple, pour opérer une rotation, il faut préciser le sens de la rotation et le degré de la rotation) et que ses préconditions soient remplies (par exemple, pour afficher les données, il faut que le fichier soit chargé, i.e. fichier chargé est une pré-condition de la commande <afficher données >).

Les sujets disposent d'un quart d'heure pour résoudre chaque tâche.

\subsection{2.- La sélection des participants}

Huit sujets ont participé à l'expérience, ce sont des étudiants en $2^{\text {m̀me }}$ année de géologie.

Les deux compères, chargés de simuler la machine, sont doctorants en géologie et experts du logiciel; ils ont travaillé en alternance (toutes les demi-heures).

\subsection{3.- Les consignes}

\section{Les consignes communiquées aux sujets}

Nous leur disons qu'ils sont là pour tester, évaluer un prototype. C'est une interface intelligente. Le mode d'interaction est l'oral et les échanges s'opèrent grâce à un système de micro et de hautparleur; nous leur demandons alors de produire leurs commandes oralement. Nous leur demandons de résoudre quatre tâches logicielles et de ne prendre qu'un quart d'heure pour la résolution de chacune d'elles. Pour chacune des tâches, nous leur transmettons une feuille mentionnant le nom du fichier à charger, l'état final à obtenir et quelques indications sur certaines commandes à effectuer. Nous les informons qu'après cette résolution, nous nous entretiendrons avec eux pour qu'ils puissent exprimer leurs sentiments, émettre un avis et proposer des pistes d'amélioration.

\section{Les consignes communiquées aux compères}

Les compères sont impliqués dans le projet GOCAD. Ils ont pour consigne de simuler le comportement d'une machine. Ils doivent exécuter les commandes orales des sujets et les aider s'ils estiment que c'est nécessaire (par cette formule nous induisons une interaction tutorielle a-didactique: le but n'est pas de faire apprendre, mais d'aider à la résolution). Nous leur demandons également lorsque l'exécution d'une commande prend du temps, de l'indiquer au sujet pour maintenir le canal de communication.

\section{2.- La dimension relationnelle de l'interaction GOCAD}

\subsection{1.- Contrats de communication externes}

C'est par le biais des consignes que nous communiquons des indications sur le contrat de communication externe. Les consignes transmises aux sujets et aux compères n'étant pas similaires, les contrats de communication externes pour chacun d'eux diffèrent quelque peu. 


\section{Contrat de communication externe des sujets}

Il comporte des indications:

- Sur la nature de l'interaction: une interaction orale homme-machine, une interaction finalisée (quatre tâches sont à résoudre);

- Sur les rôles: eux vont devoir énoncer des commandes et donc la machine va devoir les exécuter;

- Sur la manière dont les rôles vont se réaliser: la machine est dite « intelligente », elle devait donc se comporter comme une machine intelligente.

\section{Contrat de communication externe des compères}

Il comporte des indications :

- Sur la nature de l'interaction: une interaction orale homme-machine, une interaction finalisée (quatre tâches sont à résoudre), une interaction tutorielle a-didactique (les sujets ne sont pas en situation d'apprentissage);

- Sur les rôles: les sujets vont énoncer les commandes et eux, vont les exécuter, ils vont informer les sujets sur l'état de la tâche, ils vont les aider si nécessaire, ils s'attendent donc à ce que les sujets ponctuellement éprouvent des difficultés;

- Sur la manière d'accomplir leurs rôles : ils doivent se comporter à la manière d'une machine intelligente capable d'assister si besoin.

\subsection{2.- Le contrat de communication coconstruit}

Cerner le contrat de communication coconstruit consiste à étudier la transcription des interactions produites afin d'identifier:

- Les rôles

- Les procédés langagiers utilisés pour accomplir les rôles

- L'axe de la relation verticale (les relations hiérarchiques)

- L'axe de la relation horizontale (la proximité/distance entre les interlocuteurs)

Nous restituons à la suite les résultats de cette analyse.

\subsubsection{1.- La détermination des rôles}

Les compères

- exécutent les commandes: cela renvoie aux actions logicielles réalisées

- décrivent l'état courant: ce sont les interventions qui décrivent l'état de la tâche: «opération en cours » « opération effectuée » « surface interpolée »...

- expliquent : ce sont les interventions qui communiquent les raisons pour lesquelles une commande de ne peut être exécutée « commande incompréhensible» «commande impossible à exécuter à ce niveau de résolution $» . .$.

- définissent : ce sont des interventions qui communiquent des définitions d'objet (cage, dôme de sel...) ou de commande (mailler, interpoler...)

- assistent : ce sont des interventions qui participent à rendre exécutables des commandes, en faisant en sorte qu'elles soient complètes et/ou que leur pré-condition soit remplie

- informent: ce sont des interventions qui informent sur la terminologie du domaine de l'application - le lexique à employer pour la formulation des commandes

— guident: sont des interventions qui permettent de poursuivre la résolution lorsque le sujet ne sait plus quoi faire

Le premier rôle est le rôle d'exécutant prescrit dans le contrat via les consignes communiquées, le 
second est également un rôle prescrit qui avait pour but le maintien du canal de communication; les quatre suivants correspondent au rôle d'assistant à propos duquel nous étions restés flous.

Les sujets

- ordonnent : ce sont des interventions par lesquelles des actions logicielles (commandes) sont ordonnées

— informent: ce sont des interventions qui communiquent des informations sur les attributs de commandes

- acceptent : ce sont des interventions par lesquelles des suggestions sont acceptées

- questionnent: ce sont des interventions par lesquelles sont demandées des informations sur des objets de l'application et sur des commandes (demande de définition ou demande d'information sur la procédure)

- remercient : ce sont des interventions par lesquelles s'opèrent des remerciements.

Le premier rôle est celui explicitement formulé via les consignes. Les deux suivants ont été suscités par l'activité d'assistance des compères et le troisième a notamment été induit par l'adjectif «intelligent » que nous avions utilisé pour qualifier la machine au cours de l'entrevue avec les sujets. Le dernier rôle ne s'est produit que quelques fois.

\subsubsection{2.- Les procédés langagiers pour accomplir les rôles}

Puisqu'il s'agit d'accéder à des procédés langagiers, il nous faut faire abstraction de l'aspect sémantique des commandes (ne pas distinguer la commande «mailler», de la commande « interpoler » ou de la commande « affiner »...). En revanche, pour pouvoir rendre compte de l'articulation des composants du discours, il est essentiel au niveau du contenu propositionnel de pouvoir distinguer dans une séquence la présence de deux commandes différentes, de pouvoir distinguer les états de choses que chacune d'elles va générer et de pouvoir établir un lien entre la commande, l'état de choses résultant de sa réalisation et l'action logicielle qui correspond à la réalisation de celle-ci.

Nous notons les commandes représentées dans le contenu propositionnel des énoncés : (a, b, c,); les états de choses, (a', b', c',...) ; les définitions d'action (a",...) d'objets (o") et nous représentons les actions logicielles entre crochets $\langle a, b, c, \ldots\rangle$. Ainsi, (a) est une commande produite dans un énoncé, (a') est l'état de choses résultant de la commande (a) également restitué dans un énoncé et $<a>$ renvoie à la réalisation logicielle de la commande (a); (a") est la définition de l'action ou fournit des informations sur cette action.

Les négations sont notées: $\neg$

Les forces illocutoires distinguées:

O renvoie à une requête d'action: le locuteur demande que l'allocutaire réalise quelque chose (par exemple le sujet énonce: « faire un maillage »);

$\mathrm{R}$ renvoie à une requête d'information: le locuteur demande que l'allocutaire lui communique une information (par exemple le sujet énonce: « qu'est-ce qu'une cage?»);

$\mathrm{A}$, à une assertion: le locuteur décrit un état de choses (par exemple, le compère énonce: " maillage effectué »);

C, à un commissif: le locuteur s'engage à faire quelque chose (par exemple, le compère énonce: « je réalise le maillage »);

E, à un expressif: le locuteur exprime son état psychologique (le sujet énonce: «merci »).

$\mathrm{x} \rightarrow$, renvoie à une demande d'accord (par exemple, le compère énonce : « désirez-vous...»);

$\mathrm{x}$, à un accord (par exemple, le sujet énonce: « oui »).

Pour cette analyse et également pour l'étude de l'axe de la relation verticale, nous nous référons aux lois logiques qui articulent les conditions de réussite et de satisfaction des actes de langage (Trognon, 
\& Brassac, 1992). Une requête d'action est dite satisfaite lorsque son contenu est devenu vrai du fait de sa direction d'ajustement. Si un acte directif est satisfait, cela signifie qu'il est réussi (il était compréhensible et satisfaisable en contexte). Si un acte n'est pas satisfait, c'est qu'il n'a pas été compris ou que certains de ses composants n'étaient pas obtenus en situation. Dans le contexte GOCAD, une requête d'action peut ne pas être satisfaite pour différentes raisons: elle n'est pas comprise, le système n'a pas les capacités pour la satisfaire, elle est incomplète, une ou plusieurs de ses pré-conditions ne sont pas remplies.

Pour chaque rôle, nous précisons ses circonstances d'apparition, nous formalisons son accomplissement séquentiel ( $\mathrm{S}=$ sujet; $\mathrm{C}=$ =compère) suivi d'une illustration, puis nous décrivons sa forme et sa fonction interlocutoire.

\section{LES COMPÈRES}

- exécutent les commandes: cela renvoie aux actions logicielles réalisées

Circonstances d'apparition:

Les compères occupent ce rôle, i) lorsque le sujet vient de produire une requête d'action satisfaisable en contexte, ii) suite à un accord du sujet faisant suite à une demande d'autorisation pour l'exécution d'une commande.

\section{Inscription séquentielle:}

\begin{tabular}{|c|c|c|}
\hline J & $\mathrm{O}(\mathrm{a})$ & « contraindre la surface » \\
\hline C & $<\mathrm{a}>$ & $<$ contrainte de la surface $>$ \\
\hline & $\mathrm{A}\left(\mathrm{a}^{\prime}\right)$ & « surface contrainte » \\
\hline & $\mathrm{x} \rightarrow \mathrm{C}(\mathrm{a})$ & « désirez-vous que je contraigne la surface?» \\
\hline & $\mathrm{X}$ & « oui $»$ \\
\hline & $<\mathrm{a}>$ & $<$ contrainte de la surface $>$ \\
\hline & $\mathrm{A}\left(\mathrm{a}^{\prime}\right)$ & «surface contrainte » \\
\hline
\end{tabular}

Accomplir ce rôle, consiste à réaliser une action logicielle, en d'autres termes à effectuer une commande.

- décrivent l'état courant: interventions qui décrivent l'état de la tâche comme « opération en cours », « opération effectuée », « surface interpolée »...

\section{Circonstances d'apparition:}

Les compères occupent ce rôle, i) lorsque le sujet vient de produire une requête d'action satisfaisable en contexte, ii) suite à un accord du sujet faisant suite à une demande d'autorisation pour l'exécution d'une commande.

L'acte de langage « opération en cours » n'apparaît que lorsque l'action logicielle prend du temps. Les actes de langage tels « opération effectuée » ou «surface interpolée » apparaissent une fois la commande logicielle exécutée.

\section{Inscription séquentielle:}

\begin{tabular}{|c|c|c|}
\hline & $\mathrm{O}(\mathrm{a})$ & « contraindre la surface» \\
\hline $\mathrm{C}$ & $<a>$ & $<$ contrainte de la surface $>$ \\
\hline 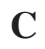 & $\mathbf{A}(\mathbf{o c})$ & «opération en cours » \\
\hline & $\mathbf{A}\left(\mathbf{a}^{\prime}\right)$ & « surface contrainte » ou « opération effectuée \\
\hline & $\mathrm{x} \rightarrow \mathrm{C}(\mathrm{a})$ & « désirez-vous que je contraigne la surface?» \\
\hline & $\mathrm{x}$ & « oui » \\
\hline
\end{tabular}


$\mathrm{C}<\mathrm{a}>$

$<$ contrainte de la surface>

C $\mathbf{A}(\mathbf{o c})$

« opération en cours »

C $\mathbf{A}\left(\mathbf{a}^{\prime}\right)$

« surface contrainte » ou « opération effectuée »

Discursivement, cela consiste par l'énonciation d'un assertif à décrire l'état de la tâche à l'écran.

- expliquent: interventions qui communiquent la raison pour laquelle une commande de ne peut être exécutée comme " commande incompréhensible», « commande impossible à exécuter à ce niveau de résolution $»$, « la surface n'est pas contrainte $» \ldots$

Circonstances d'apparition:

Les compères occupent ce rôle, i) lorsque le sujet vient de produire une requête d'action incompréhensible, ii) lorsque une ou plusieurs pré-conditions de la commande à exécuter ne sont pas obtenues dans le contexte de travail.

Inscription séquentielle:

$\mathrm{S} \quad \mathrm{O}$ ( a) $\quad$ construire la partie supérieure du dôme du diapir »

C A(inc)

« commande incompréhensible »

$\mathrm{S} \quad \mathrm{O}(\mathrm{d})$

C A(imp)

$\mathrm{S} \quad \mathrm{O}(\mathrm{b})$

« contraindre la surface»

« commande impossible à exécuter à ce niveau de résolution »

«afficher les données »

$\mathrm{S} \quad \mathrm{O}(\mathrm{b})$

« interpoler la surface »

C $\quad \mathbf{A}\left(\neg \mathbf{a}^{\prime}\right)$

« la surface n'est pas contrainte »

$\mathrm{S} \quad \mathrm{O}(\mathrm{a})$

« contraindre la surface»

Discursivement, cela consiste, au moyen d'un assertif, à informer le sujet de la raison pour laquelle sa commande ne peut être exécutée. Dans les deux premiers cas, le sujet propose à la suite une commande qui n'est pas liée à celle initialement énoncée; dans le troisième cas, le sujet énonce un ordre par lequel il demande l'exécution de la commande qui permettra d'obtenir l'état de choses mentionné comme faisant défaut dans le contexte de travail - état de choses qui constitue une pré-condition de sa commande initiale.

- définissent: interventions qui communiquent des définitions d'objet (cage, dôme de sel...) ou de commande (mailler, interpoler...)

Circonstances d'apparition:

Les compères occupent ce rôle, lorsque le sujet vient d'énoncer une requête d'information à propos d'un objet ou à propos d'une commande si celle-ci ne peut être exécutée en contexte (car au moins deux de ses pré-conditions ne sont pas obtenues dans le contexte de travail).

Inscription séquentielle:

$\mathrm{S} \quad \mathrm{R}(\mathrm{o})$ «qu'est ce qu'une cage? »

$\mathrm{C} \quad \mathrm{A}(\mathrm{o}$ ") « une cage est un parallélépipède qui entoure des objets. Cet objet permet de définir des plans de coupe »

$\mathrm{S} \quad \mathrm{R}(\mathrm{a}) \quad$ «comment faire une coupe?»

C $\quad A(a$ ") $\quad$ "pour créer une coupe, il faut générer un plan »

Discursivement, cela consiste par l'énonciation d'un assertif à satisfaire une requête d'information. 
- assistent: interventions qui participent à rendre exécutables des commandes, en faisant en sorte qu'elles soient complètes et/ou que la pré-condition faisant défaut soit remplie

Circonstances d'apparition:

Les compères occupent ce rôle, lorsque le sujet vient de produire une commande qui ne peut être satisfaite soit parce qu'elle n'est pas complète (un ou plusieurs de ses attributs ne sont pas mentionnés), soit parce qu'une de ses pré-conditions n'est pas remplie dans le contexte de travail.

\section{Inscription séquentielle:}

\begin{tabular}{|c|c|c|}
\hline $\mathrm{S}$ & $\mathrm{O}(\mathrm{c})$ & « changer la couleur de la surface» \\
\hline C & $\mathbf{R}(\mathbf{0})$ & « avec quelle couleur? » \\
\hline $\mathrm{S}$ & $\mathrm{A}\left(\mathrm{o}^{\prime}\right)$ & « rouge » \\
\hline$S$ & $\mathrm{O}(\mathrm{b})$ & « interpoler la surface» \\
\hline 0 & $\mathbf{x} \rightarrow \mathbf{C}(\mathbf{a})$ & « désirez-vous que je contraigne la surface?» \\
\hline & $\mathrm{x}$ & «oui » \\
\hline$C$ & $<a>$ & $<$ contrainte de la surface $>$ \\
\hline & $A\left(a^{\prime}\right)$ & « surface contrainte » \\
\hline & $\mathrm{C}(\mathrm{b})$ & «j'effectue l'interpolation» \\
\hline & $<b>$ & <interpolation de la surface> \\
\hline & A(b') & «surface interpolée » \\
\hline
\end{tabular}

Discursivement, participer à la complétude d'une commande revient, par une requête d'information, à questionner le ou les attributs faisant défaut; remplir la pré-condition d'une commande revient à énoncer un acte illocutoire complexe par lequel le compère demande l'autorisation pour pouvoir réaliser un commissif (en l'occurrence la commande qui permettra d'obtenir la pré-condition de la commande demandée par le sujet).

- informent: interventions qui informent sur la terminologie du domaine de l'application - le lexique à employer pour la formulation des commandes

Circonstances d'apparition:

Les compères occupent ce rôle, lorsque le sujet vient de produire une commande qui n'est pas formulée dans le langage de l'application. En fait deux cas de figure se présentent, soit la commande du sujet est formulée dans un langage qui n'est pas celui de l'application, soit la commande n'est pas tout à fait celle requise en contexte voire n'est pas non plus correctement formulée.

Inscription séquentielle:

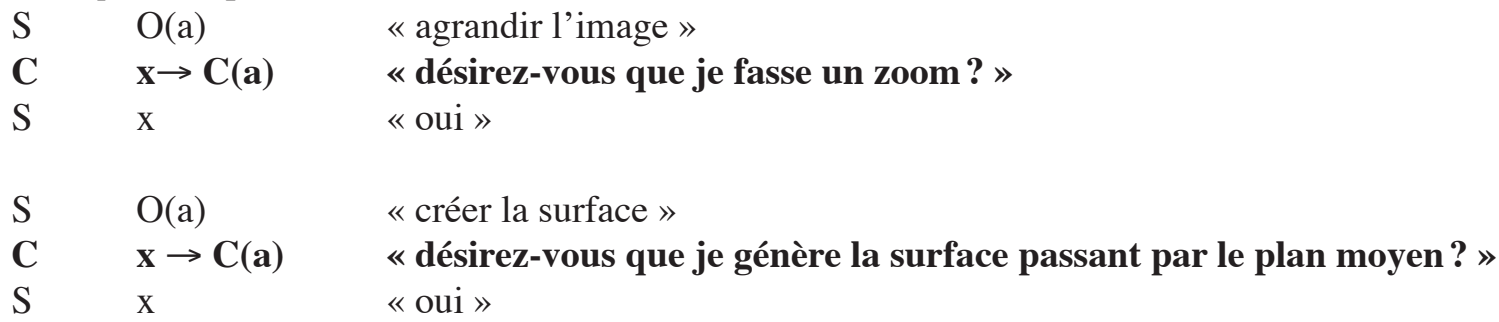

Discursivement, cela consiste à formuler la commande intentionnée par le sujet ou pertinente en contexte dans le langage de l'application par le biais d'une demande d'autorisation pour la réalisation d'un commissif.

- guident: interventions qui permettent de poursuivre la résolution lorsque le sujet ne sait plus quoi faire 


\section{Circonstances d'apparition:}

Les compères occupent ce rôle, lorsqu'un silence fait suite à l'exécution d'une commande et que l'état de choses à l'écran remplit les pré-conditions d'une autre commande.

\section{Inscription séquentielle:}

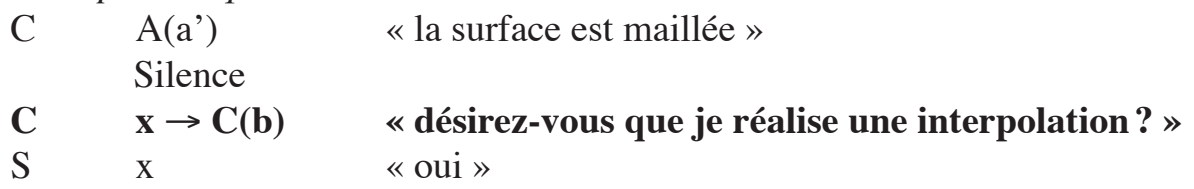

Discursivement, cela consiste à suggérer l'exécution d'une commande par le biais d'une demande d'autorisation pour la réalisation d'un commissif.

\section{LES SUJETS}

- ordonnent: interventions par lesquelles des actions logicielles (commandes) sont ordonnées

\section{Circonstances d'apparition:}

Les sujets occupent ce rôle i) en début de session lorsque le compère signifie que la session peut débuter, ii) lorsque le compère signifie qu'une commande vient d'être effectuée, iii) lorsque le compère spécifie la non existence d'un état de choses suite à une requête d'action des sujets.

\section{Inscription séquentielle:}

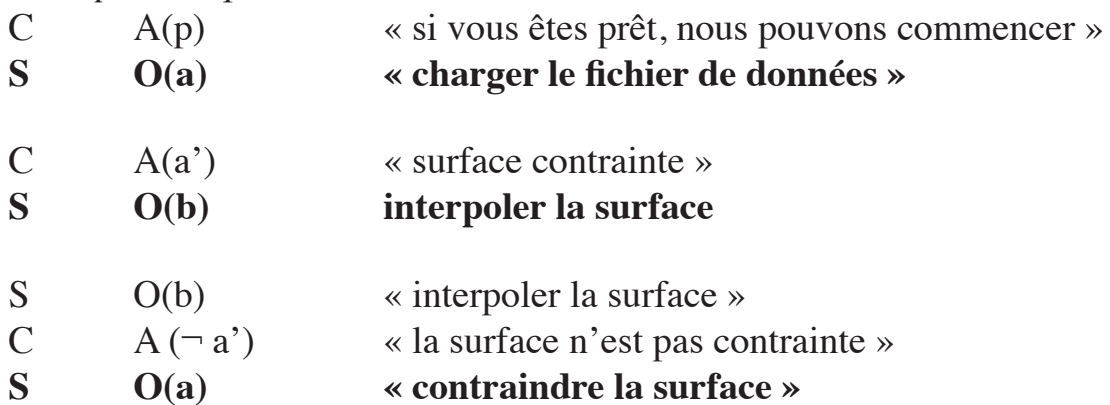

Discursivement, cela consiste à produire une requête d'action.

- informent: interventions qui communiquent des informations sur les attributs de commandes

\section{Circonstances d'apparition:}

Les sujets occupent ce rôle suite à une requête d'information du compère par laquelle il questionne un attribut d'une commande initialement incomplète.

\section{Inscription séquentielle:}

$\begin{array}{lll}\mathrm{S} & \mathrm{O}(\mathrm{a}) & \text { "faire une rotation vers la droite » } \\ \mathrm{C} & \mathrm{R}(\mathrm{o}) & \text { "de combien de degrés? » } \\ \mathbf{S} & \mathbf{A}\left(\mathbf{o}^{\prime}\right) & \text { « } \mathbf{3 0} \text { degrés } ~\end{array}$

Discursivement, cela consiste à énoncer un assertif dont le contenu satisfait la requête d'information du compère.

- acceptent: interventions par lesquelles des suggestions sont acceptées 
Circonstances d'apparition:

Les sujets occupent ce rôle, lorsque le compère demande leur accord pour réaliser une commande.

Inscription séquentielle:

$\mathrm{C} \quad \mathrm{x} \rightarrow \mathrm{C}(\mathrm{b}) \quad$ «ésirez-vous que je réalise une interpolation? »

$\mathrm{S} \quad \mathrm{x} \quad$ «oui »

Discursivement, cela consiste à produire un expressif par lequel l'accord est manifesté.

- questionnent: interventions par lesquelles sont demandées des informations sur des objets de

l'application et sur des commandes (demande de définition ou d'information sur la procédure)

Circonstances d'apparition:

Les sujets occupent ce rôle lorsque le compère signifie qu'une commande vient d'être effectuée.

Inscription séquentielle:

$\mathrm{C} \quad \mathrm{A}\left(\mathrm{a}^{\prime}\right)$ «la surface est maillée »

$\mathrm{S} \quad \mathrm{R}(\mathrm{o})$ «qu'est ce qu'un dôme? "

C A(a') «la surface est maillée »

$\mathrm{S} \quad \mathrm{R}(\mathrm{a}) \quad$ «u'est ce qu'un maillage? » ou « comment faire un maillage? »

Discursivement, cela consiste à énoncer une requête d'information à propos d'un objet ou d'une commande de l'application.

remercient : interventions par lesquelles s'opèrent des remerciements.

Circonstances d'apparition:

Ce rôle est apparu, quelques fois, lorsque le compère venait de satisfaire littéralement une demande d'information sur un objet ou une commande de l'application.

Inscription séquentielle:

$\mathrm{S} \quad \mathrm{R}(\mathrm{o}) \quad$ «qu'est ce qu'une cage? »

$\mathrm{C} \quad \mathrm{A}(\mathrm{o}$ ") $\quad$ une cage est un parallélépipède qui entoure des objets. Cet objet permet de définir des plans de coupe »

S E merci »

Discursivement, cela consiste à produire un expressif par lequel le sujet remercie son interlocuteur.

\subsubsection{3.- L'axe de la relation verticale}

\section{LES COMPÈRES}

Lorsque les compères définissent un objet ou une commande de l'application, lorsqu'ils exécutent une commande, lorsqu'ils communiquent des informations sur l'état de la tâche («opération en cours », « opération effectuée »)

La force illocutoire assertive de ces actes de langage et leur fonction réactive (dans le premier cas, les compères réagissent à une requête d'information des sujets; dans les deux autres, à une requête d'action des sujets) placent les compères en position basse et corrélativement les sujets en position 
haute.

Aussi, par ces actes de langage, les compères ne font pas que réagir à des actions des sujets, mais ils satisfont leur demande (en effectuant la commande ou en communiquant la définition) ou communiquent leur intention de satisfaction («opération en cours ») ou encore rendent compte de la satisfaction effectuée («opération effectuée »). Ainsi, les compères manifestent qu'ils se soumettent aux exigences des sujets et de fait accentuent la position haute des sujets.

Lorsque les compères énoncent que la requête d'action des sujets n'est pas satisfaisable (la commande n'est pas compréhensible, la commande n'est pas exécutable en contexte, un état de choses n'est pas obtenu)

Les actes des compères sont réactifs et leur force illocutoire assertive (des taxèmes de position basse pour le locuteur). Mais, signalons que la suite préférée d'une requête est sa satisfaction (Levinson, 1983). Or ici, par ces actes de langage, les compères communiquent qu'ils ne satisfont pas la requête des sujets. En outre, ces actes de langage rappellent l'asymétrie cognitive des partenaires (un sujet novice qui ne sait pas grand-chose de l'application et une machine experte). Ces éléments conduisent alors à envisager un rapport de places en défaveur des sujets.

Toutefois, notons que les compères tentent d'atténuer la position basse des sujets. Ils ne font pas que signifier la non satisfaction; ils en expliquent les raisons. En effet, les compères communiquent implicitement qu'ils n'opèrent pas une non satisfaction parce qu'ils s'en donnent le pouvoir mais parce que les contraintes de l'application sont telles qu'ils ne peuvent faire autrement; justifications qui contribuent à atténuer la position basse des sujets.

\section{Lorsque les compères questionnent les sujets pour compléter une commande}

Dans ce cas de figure, la requête d'action des sujets est suspendue, ce qui n'est pas une suite préférée. Toutefois cette suspension a pour but d'initier un travail sur les conditions de réussite de la requête d'action des sujets. Ainsi, le projet des compères qui transparaît dans le discours, est de faire en sorte qu'ils puissent ultérieurement satisfaire la requête d'action des sujets, ce qui les conduira à arborer une position basse et corrélativement à doter les sujets d'une position haute.

Il semble d'ailleurs que la position dans laquelle se trouvent les sujets ne soit pas très inconfortable puisque nous observons que les sujets continuent au cours des sessions à produire des commandes incomplètes alors qu'ils ont eu connaissance des attributs à fixer pour qu'elles soient d'emblée satisfaisables.

\section{Lorsque les compères suggèrent une commande visant à remplir la pré-condition d'une com- mande demandée par les sujets}

Par cet acte, ils suspendent la requête d'action des sujets; ce qui n'est pas une suite préférée. Les sujets se trouvent alors dans une position plutôt basse, accentuée par le fait que cette non satisfaction met en exergue une erreur des sujets et donc rappelle l'asymétrie cognitive en défaveur des sujets. Toutefois le procédé utilisé par les compères pour suggérer cette commande va permettre de rehausser la position basse des sujets. Ce procédé consiste à doter le commissif $\mathrm{C}(\mathrm{b})$ d'une condition préparatoire qui se rapporte aux états mentaux des sujets : pour que le commissif soit satisfait, il faut - c'est une condition nécessaire - que les sujets donnent leur accord, qu'ils expriment le désir que le commissif soit satisfait. Et c'est sur cette demande d'autorisation, que l'enchaînement va s'opérer. Donc, ce qui est mis en avant dans l'espace de l'interlocution, c'est le pouvoir qu'ont les sujets sur le processus de corésolution et moins leur incapacité à produire des commandes satisfaisables.

\section{Lorsque les compères suggèrent de réaliser une commande à la suite d'un silence}


Cette fois-ci, la fonction de l'acte est initiative, ce qui constitue un taxème de position haute. Mais comme précédemment, les compères pour atténuer la position haute dans laquelle ils se trouvent et donc simultanément atténuer la position basse des sujets, dotent leur commissif d'une condition préparatoire: avoir l'accord des sujets; ce qui permet de mettre en exergue que ce sont les sujets qui ont le pouvoir sur la progression de la résolution.

\section{LES SUJETS}

\section{Lorsque les sujets questionnent ou ordonnent}

Ils sont en position haute pour deux raisons. D'une part, leur acte de langage a une fonction initiative - ce sont les sujets qui ont le pouvoir sur la trame du discours et sur le processus de résolution. D'autre part, la force illocutoire de leur acte est directive, ce qui constitue également un taxème de position haute.

Ainsi les sujets se placent en position haute et convoquent simultanément leur partenaire à une position basse. Notons que les sujets n'utilisent aucun procédé visant à atténuer la dissymétrie des positions.

\section{Lorsque les sujets informent ou acceptent}

Dans les deux cas de figure, leur acte est réactif (réaction à une demande d'information dans le premier cas et à une demande d'autorisation dans le second); leur position est de fait plutôt basse. Toutefois dans les deux cas, leur acte a pour but de rendre satisfaisable leur propre requête d'action, dont la satisfaction aura pour conséquence de les placer en position haute. Et aussi, dans le second cas, satisfaire une demande d'autorisation, c'est également faire exister dans l'espace de l'interlocution, leur pouvoir sur la conduite de la résolution, ce qui contribue à rehausser leur position basse.

\section{Lorsque les sujets remercient}

Ils se placent eux-mêmes en situation basse et placent les compères en position haute.

\subsubsection{4.- L'axe de la relation horizontale}

Il s'agit ici d'identifier des taxèmes qui vont instruire le degré de proximité entre les partenaires, tels le style utilisé, le choix terminologique, les structures phrastiques...

Les sujets ont utilisé des formes phrastiques simples (sujet - verbe - complément) voire (verbe complément). L'emploi de connecteurs logiques est très rare. Les sujets ne participent pas aux rituels d'ouverture (à l'exception d'un sujet - nous évoquons ce cas dans le paragraphe suivant) et de fermeture. On note une quasi-absence des formules de politesse.

Ces résultats vont dans le sens des travaux en DHM pour caractériser le comportement des utilisateurs.

Si l'on s'intéresse maintenant aux modes grammaticaux utilisés par les sujets pour s'adresser à la machine lors de l'énonciation de requêtes d'action, on observe que le mode infinitif est le plus employé («faire un maillage »). Il est utilisé systématiquement par cinq sujets. On note chez les trois autres sujets, des formules d'adressage indirect via le conditionnel présent à la première personne (« je voudrais mailler la surface ») ou le présent de l'indicatif à la première personne (« je désire faire un maillage »). Parmi ces trois sujets, deux sujets ont ponctuellement vouvoyé la machine; l'un, en utilisant le présent de l'indicatif à la deuxième personne du pluriel («pouvez-vous faire un maillage? »), l'autre, en utilisant le présent de l'impératif à la deuxième personne du pluriel («faites un maillage »). Le troisième a tutoyé la machine. Précisons que l'entretien post-expérience a permis de confirmer que ce sujet avait cru interagir avec une machine. L'analyse du corpus a révélé que 
l'initiation de l'interaction avec ce sujet avait été différente des autres. Si pour les autres sessions, les compères ont initié par «si vous êtes prêts nous pouvons commencer »; avec ce sujet, les compères ont débuté par: «bonjour comment vous appelez-vous? ». Ce procédé a conduit à une plus grande proximité entre les partenaires qui sans doute explique le choix du tutoiement par ce sujet.

De leur côté, les compères ont vouvoyé les sujets ; ils ont utilisé des actes illocutoires complexes de la forme $\mathrm{x} \rightarrow \mathrm{C}$ (a) (« désirez-vous que je réalise un maillage »); ils ont utilisé des formules et des procédures langagières répétitives ( « opération en cours », « opération effectuée », « désirez-vous... »); ils n'ont pas géré les anaphores; ils ont utilisé une tonalité a-prosodique. Leur activité discursive révèle qu'ils imputent aux sujets les croyances suivantes: les systèmes fonctionnant en langue naturelle orale ne maîtrisent pas encore tout à fait la prosodie, ils ne gèrent pas les anaphores et produisent des formules répétitives. Ces caractéristiques renvoient aux subterfuges utilisés par les compères pour faire croire aux sujets qu'ils avaient affaire à une machine.

\subsection{3.- La satisfaction des sujets}

À la suite des sessions, les sujets ont été interviewés. Nous leur avons posé deux questions. Qu'avezvous pensé de l'interface orale? Est-ce que le système d'aide était suffisant, efficace?

\subsubsection{1.- L'interface orale}

Les sujets pour la plupart ont souligné que l'interaction en langue naturelle est plus intéressante que les interfaces classiques avec menus: «c'est plus facile de poser des questions, il est efficace et pertinent... on n'a pas à chercher cent sept ans dans un menu.. », « ça va beaucoup plus vite de ne pas avoir de menu.. », «j'ai parlé naturellement, j'ai bien été compris ».

Toutefois des sujets notent que ce mode d'interaction ne permet qu'une appropriation tardive des capacités de l'interface et des fonctionnalités du logiciel: « c'est une machine, on ne sait pas quel mot employer », « ce qui manquait, c'est une idée des capacités du système ».

Ce qui révèle qu'en amont de l'utilisation du système à concevoir, il serait sans doute nécessaire de présenter davantage le fonctionnement du système pour que les sujets puissent invoquer un contrat de communication externe plus précis - l'analyse du corpus fournit les ressources pour pouvoir le faire.

De nombreux sujets signalent un manque de convivialité, d' humanité: «c'est un peu sec, rechercher... comme une voix humaine, c'est un peu moins froid, impersonnel », «c'est pas très convivial (...) le langage c'est quelque chose, la voix c'est autre chose, c'est pas un problème, on pourrait choisir sa voix (...) un système qui parlerait comme un humain parle $(. .$.$) , il faudrait faire en sorte que le$ langage soit plus convivial $(. .) »$, « mais le retour vocal va devenir assez rapidement monotone ».

Ce que critiquent les sujets ce sont finalement les subterfuges utilisés par les compères pour faire croire en la véracité de l'interaction homme-machine.

\subsubsection{Le système d'aide}

Les sujets ont dit qu'ils étaient satisfaits du système d'aide, qu'ils l'avaient trouvé suffisant.

À travers leur propos, on note que certains sujets ont cerné les différentes formes d'assistance «il oriente.., si on a un trou, il pose une question, une opération qui serait logique de faire ». Deux sujets n'ont perçu l'aide que lorsque ce sont eux qui en ont fait explicitement la demande, c'est-à-dire lorsqu'ils ont produit des requêtes d'information: « je ne m'en suis pas trop servi, mais quand je m'en suis servi ça a bien marché », «l'aide je l'ai utilisée que deux ou trois fois, elle m'est apparue sûre car il a répondu à mes questions ».

Ainsi, les sujets sont davantage intervenus sur l'axe de la relation horizontale en demandant d'humaniser l'interface; l'axe de la relation verticale n'a pas outre mesure affecté leur sentiment de satisfaction. 


\section{3.- Discussion}

\subsection{1.- Une interaction fonctionnelle}

Rappelons que l'enjeu de l'interaction est la résolution de quatre tâches. Résoudre des tâches avec le logiciel GOCAD consiste à exécuter dans un ordre adéquat une succession de commandes.

Dans le cadre de cette expérience, une dizaine de commandes était nécessaire pour la résolution de chaque tâche, d'autres pouvaient être exécutées pour améliorer le résultat graphique à l'écran.

Nous observons qu'en moyenne 13, 41 commandes ont été exécutées par tâche avec un écart-type de 3,19 .

Globalement les résultats obtenus par chaque dyade sont satisfaisants.

La première tâche a été résolue entièrement par six dyades; pour une dyade, il manquait une commande à effectuer; pour la dernière, deux commandes.

La deuxième tâche a été résolue entièrement par trois dyades; pour deux dyades, il manquait deux commandes; pour trois dyades, il manquait quatre commandes.

La troisième tâche a été résolue entièrement par cinq dyades; pour les trois autres, il manquait une commande à effectuer.

La quatrième tâche a été résolue entièrement par les huit dyades.

\subsection{2.- Une interaction homme-machine}

L'étude des entretiens et du corpus permet de confirmer que le corpus présente bien les caractéristiques des DHM.

Les propos tenus en entretien présupposent que les sujets ont cru interagir avec une machine : «on se rend compte qu'il y a plein de choses qu'elle comprend »...;

La structure phrastique des énoncés des sujets, la façon dont ils s'adressent à leur interlocuteur, la non participation aux rituels d'ouverture, la quasi absence de formules de politesse... sont typiques des DHM.

Aussi l'étude de la relation verticale a révélé que les sujets ne cherchaient pas à atténuer la position basse de leur partenaire. En effet, lorsque les compères occupent une place d'exécutant et donc sont en position basse, les sujets n'emploient aucun procédé permettant de rehausser leur position basse. Ce qui montre là aussi que les sujets ont cru avoir affaire à un artefact qui n'a pas de face; dans ces circonstances, il était donc inutile de déployer des procédés ou processus de figuration dans la terminologie de Goffman (1974) visant à préserver sa face.

\subsection{3.- Une interaction tutorielle}

L'asymétrie cognitive s'est bien révélée. Des connaissances sur le domaine d'application que les sujets ne connaissaient pas, ont bien été communiquées par les compères. Ce sont des connaissances terminologiques (commandes formulées dans le langage de l'application), des connaissances sur les attributs des commandes, sur leurs pré-conditions, des définitions d'objets ou de commandes de l'application.

Ces connaissances ont été produites au cours des résolutions et induites par des lacunes des sujets; lacunes qui ont pris la forme de requêtes d'action qui n'étaient pas satisfaisables en contexte (commande incomplète, commande dont une pré-condition n'était pas remplie), de requêtes d'information ou encore qui se sont matérialisées par des silences.

Nous avons donc bien affaire à une interaction tutorielle. 


\subsection{4.- Une interaction a-didactique}

Nous allons restituer les éléments qui révèlent le façonnement d'un contrat de communication a-didactique.

- Ce sont les sujets qui ont le pouvoir sur la progression de la résolution. Ce sont eux qui principalement initient les séquences d'action; aussi l'exécution de toute commande nécessite leur approbation.

- C'est l'action qui importe et moins l'apprentissage. Les connaissances sont communiquées au service de l'action, elles sont communiquées pour rendre les commandes des sujets satisfaisables et donc pouvoir les exécuter. Le comportement des compères qui consiste à suggérer l'exécution d'une commande lorsque celle-ci est questionnée via une requête d'information et qu'elle est exécutable en contexte, reflète bien de cette intention d'action.

- La position basse des sujets a été atténuée. Les compères dans les circonstances où les sujets allaient se trouver en position basse, ont utilisé des procédés visant à rehausser la position basse des sujets.

- La nature des connaissances n'est pas explicitement communiquée. Les compères ne disent pas « ceci est l'attribut de la commande », « ceci est une pré-condition de la commande », etc. Les connaissances sont produites au service de l'action et moins dans une optique didactique - leur nature n'étant pas explicitement communiquée. Ce n'est que par une étude rétrospective séquentielle des actes produits que la nature de certaines connaissances se révèle. Prenons un exemple. Le sujet énonce une requête d'action et à la suite, le compère lui demande son autorisation pour réaliser une autre commande. À ce niveau, au moins deux interprétations sont envisageables : soit le compère propose une commande plus appropriée, soit il propose de remplir une pré-condition de la commande demandée par le sujet. Si le compère, après avoir exécuté la commande qu'il a suggérée, énonce qu'il va alors exécuter la commande initiale du sujet, alors à ce niveau, il sera possible de déduire que la commande suggérée par le compère va permettre d'obtenir un état de choses qui remplira une pré-condition de la commande du sujet. C'est donc la satisfaction de la commande initiale qui va permettre de déduire que l'état de choses obtenu via la satisfaction de la commande suggérée est une pré-condition de la commande initiale. Si à la suite de l'exécution de la commande suggérée, le compère ne propose pas de satisfaire la commande initiale, alors la commande suggérée par le compère sera considérée comme une commande venant se substituer à la commande initiale car plus appropriée en contexte que celle du sujet. C'est donc dans l'après-coup qu'il est possible d'identifier la nature des connaissances communiquées. Mais quelques fois, l'engendrement séquentiel ne suffit pas à cette détermination. Prenons un autre exemple. Une commande vient d'être exécutée et à la suite, le sujet ne prend pas la parole, ce qui se traduit par un silence. De fait, le compère intervient pour suggérer l'exécution d'une commande. Dans ce cas de figure, il n'est pas possible de savoir si l'état de choses résultant de l'exécution de la commande précédente constitue une pré-condition de la commande suggérée par le compère: l'état de choses peut i) être indépendant de la commande, ii) être suffisant à la réalisation de la commande ou iii) être nécessaire à son exécution (De Almeida, \& Saint-Dizier de Almeida, 1998) - c'est uniquement dans le dernier cas, que l'état de choses est une pré-condition de la commande suggérée.

- On n'observe pas de stratégies pédagogiques visant par exemple à sanctionner l'acquisition des sujets, à les placer dans des situations où ils devraient fournir des efforts pour dépasser leurs erreurs.... Dans la situation qui nous occupe, pour que l'interaction fonctionne, le coût cognitif pour le sujet est minimal; il suffit que le sujet réponde aux questionnements et qu'il fasse confiance au système en acceptant ses suggestions.

- Toutefois, les compères ont communiqué des connaissances additionnelles. Cet élément va à l'encontre de la dimension a-didactique de la relation. Les connaissances additionnelles sont « des connaissances qui vont au-delà du besoin immédiat des opérateurs » (Falzon, \& Pasqualetti, 2000 op.cit., p. 124). Ce rôle apparaît lorsque le sujet à l'intention de faire exécuter une commande, que 
le compère accède à l'intention mais que la formulation utilisée par le sujet pour représenter le contenu de la commande est éloignée de la terminologie de l'application. Dans ces circonstances, les compères au tour suivant, énoncent un acte illocutoire complexe, par lequel ils demandent au sujet l'autorisation de réaliser la commande dont le contenu est cette fois exprimé dans le langage de l'application. Notons que cette connaissance n'est pas nécessaire à la poursuite de la résolution. Les compères ayant accédé à l'intention du sujet via l'état de la tâche et l'acte produit par le sujet, ils pourraient très bien poursuivre en procédant directement à l'exécution de la commande en question. Ils ne le font pas. Nous avons donc ici affaire à une connaissance additionnelle; les connaissances terminologiques en font partie (Falzon, \& Pasqualetti, 2000 op.cit.).

Les différentes informations mentionnées, à l'exception du dernier point, nous conduisent à approuver la validité de notre corpus: nous sommes parvenus à simuler une interaction homme-machine tutorielle a-didactique. La situation mise en place, les consignes communiquées ont donc permis d'induire un contrat de communication externe qui a contribué en situation au façonnement d'un contrat de communication correspondant à nos souhaits; elles constituent de fait des indications pour les chercheurs ou professionnels souhaitant créer ce type de situation tutorielle.

\section{5.- Conclusion}

L'étude de la dimension relationnelle des communications proposée repose sur d'une part l'identification du ou des contrat(s) de communication externe(s) et d'autre part, l'identification du contrat de communication coconstruit. L'étude consiste à identifier les rôles attendus et les rôles accomplis, la manière dont ils devraient s'accomplir et la façon dont ils se sont accomplis, la nature de la relation attendue et celle accomplie.

Le contrat de communication externe s'instruit par des méthodologies comme l'étude documentaire, les entretiens; l'identification du contrat de communication coconstruit repose sur l'analyse des communications produites. Pour l'analyse des communications nous avons recours à la logique interlocutoire. Si celle-ci est utilisée dans le domaine du travail pour restituer des processus de mise en partage de connaissances (Grusenmeyer, \& Trognon, 1997 ; Trognon, \& Saint-Dizier, 1999; Kostulski, \& Trognon, 2000), de construction de concept (Kostulski, \& Prot, 2004), pour restituer des compétences (Saint-Dizier, 1997 op.cit.; Mayen, 1998; Engrand, 2002; Mayen, \& Specogna, 2005; Batt, \& Trognon, 2009) etc., elle permet ici de restituer la manière dont les rôles se sont accomplis et en outre, elle facilite l'identification de taxèmes relationnels. Les notions utilisées pour rendre compte de la dimension relationnelle, en l'occurrence les notions de contrat de communication, de rôle, de rapport de places, de position, de taxème sont empruntées essentiellement à la psychologie sociale et à la linguistique interactionniste.

\section{L'intérêt de s'enquérir du contrat de communication externe}

L'intérêt de recourir au contrat de communication externe va dépendre de l'objectif de recherche. $\mathrm{Si}$, comme dans la recherche GOCAD, on vise la validité des données recueillies, une référence à ce qui est attendu s'impose. D'autres objectifs suggèrent également cette référence. Si l'on vise l'évaluation de la qualité d'une communication, il est indispensable de s'enquérir de ce qui est attendu. Cette référence permet d'identifier les comportements atypiques, non conventionnels et de voir s'ils contribuent à une plus grande qualité relationnelle ou si, au contraire, ils l'altèrent. La seule référence aux positions sociales occupées n'est en effet pas suffisante pour apprécier la qualité relationnelle. Même si les positions basses ne sont pas a priori recherchées, elles ne constituent pas systématiquement des indices d'une moindre qualité relationnelle. Par exemple, les positions basses occupées par les élèves en interaction tutorielle ou par les patients en entretien médical sont tout à fait acceptées par ces derniers et n'altèrent pas la qualité relationnelle, ni la satisfaction des usagers; ceci, car elles font partie du contrat de communication externe. Ainsi, l'acceptabilité des positions basses ne peut 
s'envisager qu'au regard des positions attendues.

De même, si l'on a tendance à considérer les séquences conflictuelles comme affectant la qualité relationnelle, c'est parce que la plupart des contrats de communication externes ne comportent pas ce type d'échanges orageux. Mais, imaginons que l'on ait affaire à un débat politique; dans ce cadre, ce seraient les séquences consensuelles qui seraient jugées comme inadéquates ou surprenantes et non les séquences conflictuelles. Partant, appréhender la qualité relationnelle suppose une référence à ce qui est attendu; cet attendu, nous le matérialisons à travers la notion de contrat de communication externe.

\section{L'intérêt d'investir la dimension relationnelle des communications}

D'un point de vue épistémologique, la compréhension de la dynamique des activités suppose que la dimension relationnelle soit appréhendée au même titre que la dimension opératoire parce que toutes deux sont constitutives du processus interactionnel.

Ce versant relationnel mérite également d'être investi pour des raisons davantage conjoncturelles.

- Pour améliorer les interfaces homme-machine. Dans le champ de l'ergonomie des interfaces homme-machine, les chercheurs sous l'impulsion notamment de Sansonnet (LIMSI) travaillent actuellement à améliorer la qualité relationnelle des interfaces en y intégrant des Agents Conversationnels Animés. On cherche à humaniser davantage les interfaces homme-machine pour accroître la satisfaction des usagers. On voit dès lors l'intérêt d'étudier finement cette dimension; d'autant que les travaux de Dubois et Tajariol (2003) ont montré que dans ce contexte d'interaction, la dimension relationnelle avait également une incidence sur la persévérance des utilisateurs.

- Pour comprendre les situations de mal-être au travail et agir en conséquence. Les travaux de Grosjean et Ribert-Van de Weerdt (2005) ont montré que le mal-être au travail pouvait être causé par le niveau opératoire (trop de surveillance, surcharge de travail...) mais également par le niveau relationnel (occuper des rôles que l'on ne devrait pas avoir à occuper, gérer le stress des usagers...). Rapporter le mal-être à des faits empiriques pouvant relever de la dimension relationnelle, suppose donc que cette dimension soit investie minutieusement.

- Pour améliorer la qualité des relations de service. Dans le champ des relations de service, «La qualité relationnelle de l'interaction est un critère en soi, indépendamment de la qualité des autres niveaux » (Falzon, \& Cerf, 2005 op.cit.,p.55). À prestation égale, c'est la qualité relationnelle du service qui fait la différence (ibid.). On comprend alors que l'étude de cette dimension s'avère cruciale pour les sociétés des services qui œuvrent à la fidélisation de leur clientèle.

- Pour former à la gestion du relationnel. Notamment dans le cadre des relations de service, les difficultés et les incapacités par les professionnels à réguler le relationnel, peuvent être source de souffrances, burn-out, suicides, erreurs, incidents... (Caroly, \& Weill-Fassina, 2007). Il s'agit alors pour les psychologues ergonomes, dans des optiques de formation, de capitaliser ce qui permet la qualité de ce travail relationnel (Pochat, \& Falzon, 2000). Le cadre d'analyse proposé dans notre article fournit une piste pour ce type d'investigation.

\section{RÉFÉRENCES}

Barcellini, F., Détienne, F., \& Burkhardt, J.-M. (2007). Conception de logiciels libres : enjeux pour l'ergonomie et rôle des utilisateurs dans le processus de conception. Actes du congrès de la SELF, pp. 43-52.

Batt, M., \& Trognon, A. (2009). Ergonomie cognitive d'une consultation génétique pour le test présymptomatique de maladie de Huntington. Psychologie du travail et des organisations, 15(1), 21-40.

Bilange, E. (1992). Dialogue personne-machine modélisation et réalisation informatique. Paris: Hermès. 
Brangier, E. (2002). Le travail conversationnel: Analyse de l'activité conversationnelle dans le « call-center» d'une compagnie d'assurance. In E. Engrand, S. Lambolez, \& A. Trognon (Eds.), Communications en situation de travail à distance (pp. 29-48). Nancy: PUN.

Bromberg, M. (2007). Plaidoyer pour une psycho-socio-pragmatique de la communication. In C. Chabrol, \& I. Orly-Louis (Eds.), Interactions communicatives et psychologie (pp. 27-42). Paris: Presses Sorbonne Nouvelle.

Brousseau, G. (1986). Fondements et méthodes de la didactique des mathématiques. Recherches en Didactique des Mathématiques, 7(2), 33-115.

Cahour, B. (2002). Décalages socio-cognitifs en réunions de conception participative. Le travail humain, 65(4), 315-337.

Cahour, B. (2006). Les affects en situation d'interaction coopérative: proposition méthodologique. Le Travail Humain, 69(4), 379-400.

Camus, O. (1999). Les interactions langagières. In J.-P. Pétard (Ed.), Psychologie sociale (pp. 259-316). Rosny: Editions Bréal.

Caroly, S. (2000). « Bonjour Monsieur... Merci, Au revoir » Comment l'étude des communications guichetier-client permet d'analyser l'activité de service pour la transformer ? Actes du Congrès de la SELF, pp. 276-285.

Caroly, S. (2004). Donner la vie à la mort. Normalisation et compétences des opérateurs funéraires. Actes du Congrès de la SELF, pp. 189-201.

Caroly, S., \& Weill-Fassina A. (2007). En quoi les différentes approches de l'activité collective des relations de services interrogent la pluralité des modèles de l'activité en ergonomie ? @ ctivités, 4(1). http://www. activites.org/v4n1/caroly-FR.pdf

Chapelier, L. (1996). Dialogue d'assistance dans une interface homme-machine multimodale. Thèse, Nancy: Université Nancy 1.

Charaudeau, P. (1983). Langage et discours. Paris: Hachette.

Charaudeau, P. (2006). Discours journalistique et positionnements énonciatifs. Frontières et dérives. SEMEN, $22,39-43$.

De Almeida, J., \& Saint-Dizier de Almeida, V. (1998). Conception assistée par le dialogue: des interactions verbales aux règles de fonctionnement du pilote d'un multi-robots. In K. Kostulski, \& A. Trognon (Eds.). Distribution des savoirs et coordination de l'action dans les équipes de travail (pp. 159-182). Nancy: PUN.

Delgoulet, C., Kerguelen, A., \& Barthe, B. (2000). Vers une analyse intégrée des communications et des actions au travail: quelles modalités de leur mise en relation? Actes du Congrès de la SELF, pp. 363-375.

Djibo, S. (2008). Contribution d'une analyse du discours à l'étude des stratégies d'actions et de régulation des agents en situation d'écoute téléphonique : l'exemple du dispositif d'urgence sociale 115. @ctivités, 5(1). http://www.activites.org/v5n1/djibo.pdf

Dubois, M., \& Tajariol, F. (2003). Les effets de l'interface personnifiée sur la persévérance dans la tâche et la qualité de l'interaction: le paradoxe du contexte social d'interaction. Le Travail Humain, 66(3), 225-252.

Engrand, E. (2002). Gestion conversationnelle d'un diagnostic médical dans un service d'écoute sur le cancer. In E. Engrand, S. Lambolez, \& A. Trognon (Eds.), Communications en situation de travail à distance (pp. 65-81). Nancy: PUN.

Falzon, P., \& Cerf, M. (2005). Le client dans la relation. In M. Cerf, \& P. Falzon (Eds). Situations de service : travailler dans l'interaction (pp. 41-59). Paris: PUF, Coll le travail humain.

Falzon, P., \& Pasqualetti, L. (2000). L'apprentissage opportuniste. In T.H. Benchekroun, \& A. Weill-Fassina (Eds.), Le travail collectif: perspectives actuelles en ergonomie (pp. 121-133). Toulouse: Octarès Editions.

Flahaut, F. (1978). La parole intermédiaire. Paris: Le Seuil. 
Ghiglione, R. (1989). Je vous ai compris ou l'analyse des discours politiques. Paris: Armand Colin.

Ghiglione, R., \& Trognon, A. (1993). Où va la pragmatique? Grenoble: PUG.

Goffman, E. (1974). Les rites d'interaction. Paris: Les éditions de Minuit.

Grosjean, V., \& Ribert-Van de Weerdt, C. (2005). Vers une psychologie ergonomique du bien-être et des émotions. Les effets du contrôle dans les centres d'appels. Le Travail Humain, 68(4), 355-378.

Grusenmeyer, C. (1996). De l'analyse des communications à celle des représentations fonctionnelles partagées. Thèse, Nancy: INRS.

Grusenmeyer, C., \& Trognon, A. (1997). Les mécanismes coopératifs en jeu dans les communications de travail: un cadre méthodologique. Le Travail Humain, 60(1), 5-31.

Kerbrat-Orecchioni, C. (1988). La notion de «place » interactionnelle ou les taxèmes, qu'est-ce que c'est que ça? In J. Cosnier, N. Gelas, \& C. Kerbrat-Orecchioni (Eds.), Echanges sur la conversation (pp. 185-198). Paris: Editions du CNRS.

Kostulski, K., \& Prot, B. (2004). L'activité conversationnelle d'un jury de Validation d'Acquis : analyse interlocutoire de la formation d'un concept potentiel. Psychologie Française, 49 (4), 425-441.

Kostulski, K., \& Trognon, A. (2000). Analyse des interactions et analyse des activités de travail: 1'exemple de la transmission dans les équipes de soin à l'hôpital. Revue de Psychologie du Travail et des Organisations, 6 (1-2), 207-225.

Levinson, S. (1983). Pragmatics. Cambridge: Cambridge University.

Maïs, Ch. (1989). L'adaptation de l'aide à l'utilisateur : aider les programmeurs occasionnels à réaliser leurs plans sous-optimaux. Thèse de doctorat de Psychologie, Université de Provence Aix-Marseille I.

Marcoccia, M. (2007). Communication électronique et rapport de places: analyse comparative de la formulation d'une requête administrative par courrier électronique et par courrier papier. SEMEN, 20. URL: http://semen.revues.org/document1075.html.

Mayen, P. (1998). Le processus d'adaptation pragmatique dans la coordination d'une relation de service. In K. Kostulski, A. Trognon (Eds.). Communications interactives dans les groupes de travail (pp. 205-233). Nancy: PUN.

Mayen, P., \& Specogna, A. (2005). Conseiller: une activité et un jeu de langage professionnels. In L. Filliettaz, \& J.P. Bronckart (Eds.), L'analyse des actions et des discours en situation de travail. Concepts, méthodes et applications (pp.99-113). Louvain: Bibliothèque des Cahiers de l'Institut de Linguistique de Louvain.

Pierrel, J.-M. (1987). Dialogue oral homme-machine. Paris: Hermès.

Pochat, A., \& Falzon, P. (2000). Quand faire c'est dire ou la reconnaissance du travail verbal dans l'activité d'accueil. Actes du Congrès de la SELF, pp. 293-303.

Rogalski, J. (1998). Concepts et méthodes d'analyse des processus de coopération dans la gestion collective d'environnements dynamiques. In K. Kostulski \& A. Trognon (Eds.), Communications interactives dans les groupes de travail (pp. 27-58). PUN: Nancy.

Saint-Dizier, V. (1995). Analyse d'interactions verbales pour la modélisation d'un système d'assistance interactif: une démarche de conception ascendante. Revue Internationale de Psychologie Sociale, 2 , 59-82.

Saint-Dizier de Almeida, V. (1997) Modélisation d'une assistance interactive pour améliorer l'accessibilité d'un logiciel. Revue Sciences et Techniques Educatives, 4(1), 13-39.

Schubauer-Léoni, M.L. (1986). Le contrat didactique: un cadre interprétatif pour comprendre les savoirs manifestés par les élèves en mathématiques. European Journal of Psychology of Education, 2, 139-153.

Sperandio, J.C., \& Letang-Figeac, C. (1986). Simulation expérimentale de dialogues oraux en communication homme-machine. Rapport GRECO-CNRS Communication parlée, Nancy. 
Suchman, L. (1987). Plans and Situated Actions - The problem of Human/Machine Communication. Cambridge: Cambridge University Press.

Trognon, A., \& Brassac, C. (1992). L'enchaînement conversationnel. Cahiers de Linguistique Française, 13, 76-107.

Trognon, A., \& Saint-Dizier, V. (1999), L'analyse conversationnelle d'un malentendu: le cas d'un dialogue tutoriel. Journal of Pragmatics, 31, 787-815.

Valléry, G. (2004). Relation de service et approche ergonomique: saisir le caractère dynamique et situé de l'activité au travers de l'analyse des interactions «agent-client». @ctivités, 1(2). http://www.activites. org/v1n2/vallery.pdf

Valléry, G., Boucheix, J.M., Leduc S., \& Cerf M (2005). Analyser les situations de service: questions de méthodes. In M. Cerf, \& P. Falzon (Eds.), Situations de service : travailler dans l'interaction (pp. 21-40). Paris: PUF Coll le travail humain.

Vion, R. (1992). La communication verbale. Paris: Hachette Supérieur.

Watzlawick, P., Beavin, J.H., \& Jackson, D.D.A. (1972). Une logique de la communication. Paris: Le Seuil.

\section{RÉSUMÉ}

Cet article traite de la dimension relationnelle des communications. Il décrit un cadre théorique et méthodologique pour l'étude de la relation d'une interaction homme-machine tutorielle a-didactique obtenue par la technique du magicien d'Oz.

Pour l'analyse du corpus, nous nous référons à la logique interlocutoire telle qu'elle a été développée par Trognon dans les années 90. Pour décrire et restituer la dimension relationnelle, nous utilisons des notions empruntées à la psychologie sociale et à la linguistique interactionniste, comme celles de contrat de communication, de rôle, de position sociale. L'analyse interlocutoire a notamment permis d'identifier les moyens langagiers par lesquels les protagonistes accomplissent leurs rôles et construisent conjointement un contrat de communication.

\section{MoTS CLÉS}

communication, activité, magicien d'Oz, interaction homme-machine, interaction tutorielle, relation sociale, contrat de communication, rôle, position sociale, analyse interlocutoire

\section{RÉFÉRENCEMENT}

Saint-Dizier de Almeida, V. (2009). La dimension relationnelle des communications : Étude d'interactions homme-machine tutorielles a-didactiques. Activités, 6 (2), pp. 75-99. http://www.activites.org/v6n2/v6n2. pdf 